\title{
Erythropoietin deficiency decreases vascular stability in mice
}

\author{
Jing Chen, Kip M. Connor, Christopher M. Aderman, and Lois E.H. Smith \\ Department of Ophthalmology, Children's Hospital Boston, Harvard Medical School, Boston, Massachusetts, USA.
}

\begin{abstract}
Erythropoietin (Epo), a hormone known to stimulate bone marrow erythrocyte production, is widely used to treat anemia in patients at risk for vascular disease. However, the effects of Epo on angiogenesis are not well defined. We studied the role of Epo in a mouse model of retinopathy characterized by oxygen-induced vascular loss followed by hypoxia-induced pathological neovascularization. Without treatment, local retinal Epo levels were suppressed during the vessel loss phase. Administration of exogenous Epo prevented both vessel dropout and subsequent hypoxia-induced neovascularization. Early use of Epo also protected against hypoxia-induced retinal neuron apoptosis. In contrast, retinal Epo mRNA levels were highly elevated during the retinopathy neovascular phase. Exogenous late Epo treatment did not protect the retina, but rather enhanced pathological neovascularization. Epo's early protective effect occurred through both systemic retinal recruitment of proangiogenic bone marrow-derived progenitor cells and activation of prosurvival NF- $\kappa B$ via Epo receptor activation on retinal vessels and neurons. Thus early retinal Epo suppression contributed to retinal vascular instability, and elevated Epo levels during the proliferation stage contributed to neovascularization and disease. Understanding the role of Epo in angiogenesis is critical to timing its intervention in patients with retinopathy or other diseases in which pathological angiogenesis plays a significant role.
\end{abstract}

\section{Introduction}

Oxygen-regulated growth factors play critical roles in regulating retinal angiogenesis in both development and disease (1). Erythropoietin (Epo), a hormone known to stimulate erythrocyte production in bone marrow, is such an oxygen-regulated pleiotropic growth factor (2). Epo is produced primarily in the kidney in response to anemia and hypoxia. Effects of local Epo production are not as well defined. Recombinant Epo is now widely used for treatment of anemia in patients with chronic kidney failure and cancer patients with chemotherapy-induced bone marrow suppression, as well as anemic patients who often have concurrent or are at risk for diabetic retinopathy (DR) and retinopathy of prematurity (ROP). Elevated levels of Epo are found in the vitreous samples of patients with proliferative DR $(3,4)$, suggesting a role of Epo in pathological retinal angiogenesis. However, the role of Epo in normal vascular stability is largely unknown. In addition, higher doses of Epo have recently been associated with increased risk of cardiovascular disease and tumor growth (5-8). Understanding the effect of Epo on vessel stability and angiogenesis is likely to benefit not only retinopathy patients but also patients with diseases such as cancer that are dependent on angiogenesis who are also often treated with Epo.

DR and ROP are blinding diseases with a hallmark of abnormal and excessive blood vessel growth that can cause retinal detachment. Paradoxically, it is early vessel loss in ROP and DR (and loss of neural retina) that initiates retinopathy. In ROP, supplemental oxygen and lack of growth factors cause vessel loss. In DR, hyperglycemia triggers vascular dropout. An inadequate blood supply resulting from early vessel loss causes tissue hypoxia, which determines the severity of subsequent pathological vessel growth.

Nonstandard abbreviations used: DR, diabetic retinopathy; EPC, endothelial progenitor cell; Epo, erythropoietin; ROP, retinopathy of prematurity.

Conflict of interest: The authors have declared that no conflict of interest exists. Citation for this article: J. Clin. Invest. 118:526-533 (2008). doi:10.1172/JCI33813.
Therefore a major goal of retinopathy treatment is to prevent vessel loss in order to prevent the devastating end stage of the disease. These interventions might promote normal vessel growth as well as inhibit pathological neovascularization. In this study we determined the effects of Epo on vessel loss, normal physiological vessel growth, as well as pathological angiogenesis by examining oxygeninduced retinopathy in the mouse eye (9).

Some evidence suggests that the role of Epo extends beyond erythrogenesis. The influence of Epo on angiogenesis and retinopathy is beginning to be defined. Epo has been found to promote endothelial cell proliferation and vessel growth (10). Epo is also a powerful cytoprotective factor that can protect both vascular cells and neurons from apoptosis (11). In the eye, Epo levels are elevated in the vitreous of patients with proliferative DR $(3,4)$, and inhibiting Epo in the proliferative phase in the mouse model of retinopathy can inhibit retinal neovascularization (4). However, since retinal vessel loss precedes neovascularization, and the severity of neovascularization is largely determined by the extent of initial vessel loss, understanding the role of Epo in the development of initial vessel loss in retinopathy is important. Studies focusing on this initial phase of the disease have been lacking. Anemia with functional Epo deficiency has been associated with an increased risk of developing retinopathy, and correction of anemia improves the risk $(12,13)$. Therefore we hypothesized that lack of Epo, a potent cytoprotective factor, may contribute to the initial vessel loss in retinopathy. If proven to be true, correction of Epo deficiency during the early phase of retinopathy could be of benefit to prevent the initial vessel loss and thereby prevent the devastating proliferative stage of the disease, whereas the use of Epo when tissue levels of Epo are high may exacerbate proliferation of vessels.

\section{Results}

Epo expression during oxygen-induced retinopathy. Epo mRNA expression from whole retinas during vessel development as well as oxygen-induced retinopathy was examined by quantitative real-time 

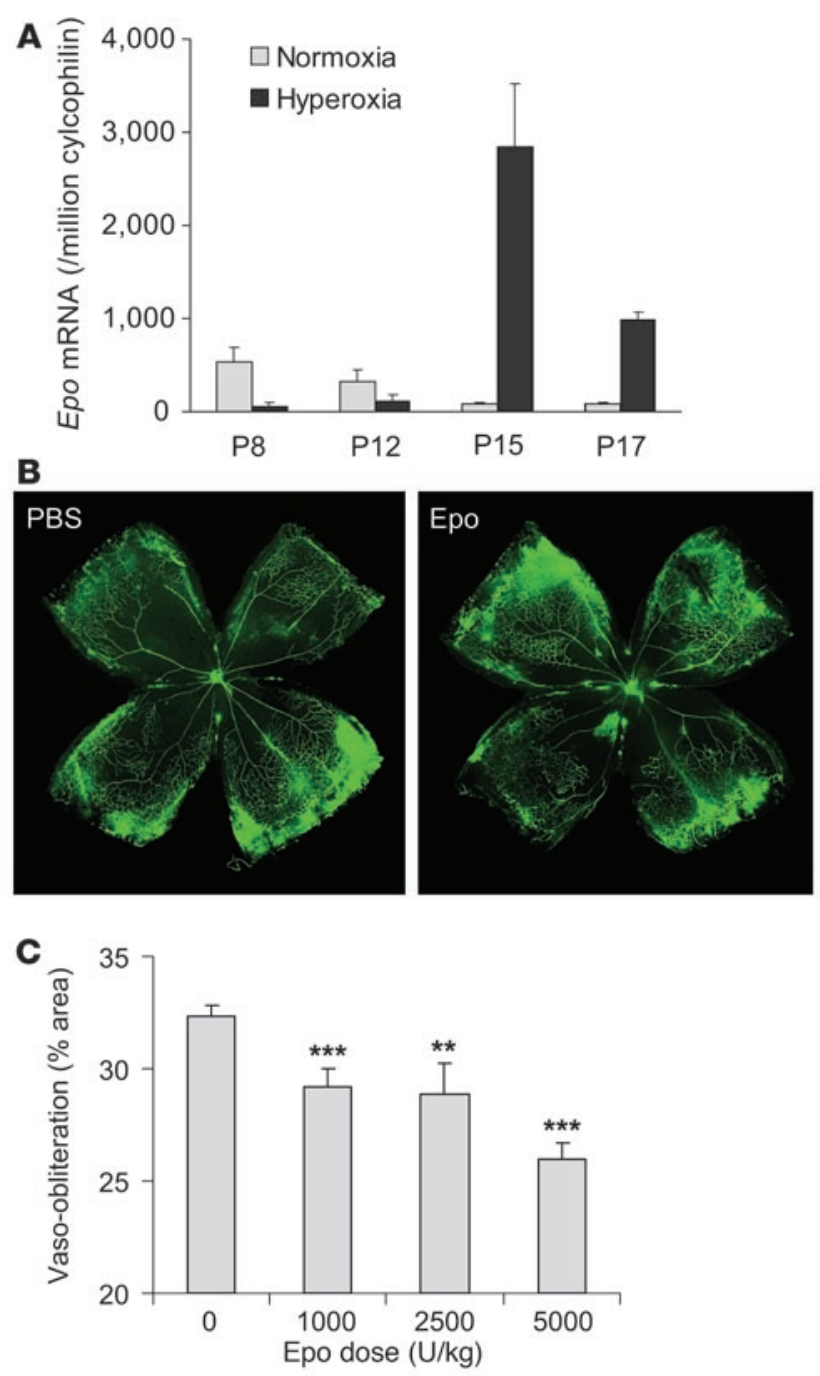

PCR. Compared with age-matched normoxia controls, retinal Epo mRNA expression was greatly suppressed (9.9-fold at P8 and 2.8-fold at P12; Figure 1A) during hyperoxia treatment (P7-P12), when oxygen-induced retinal vessel loss occurs. After returning to room air, hypoxia induces retinal neovascularization (P12-P17). During the hypoxic phase, Epo mRNA was increased dramatically (33.2-fold at P15 and 11.5-fold at P17) compared with controls (Figure 1A). These data suggest that lack of Epo in the first phase might contribute to early vessel loss and the onset of retinopathy, while the increase of Epo in the second phase might play a role in neovascularization.

Early use of exogenous Epo prevents retinal vessel loss. We next examined whether exogenous Epo treatment during hyperoxia-induced vaso-obliteration (when retinal Epo levels are suppressed) helps to prevent retinal vessel loss. At P8, mice treated with Epo (i.p., P6 and $\mathrm{P} 7,5,000 \mathrm{U} / \mathrm{kg}$ ) had a vaso-obliterated/total retinal area of $26.0 \% \pm 0.7 \%$ compared with $32.4 \% \pm 0.5 \%$ in saline-injected littermate controls $(P \leq 0.001$; Figure $1, \mathrm{~B}$ and $\mathrm{C})$, suggesting Epo treatment had a dose-dependent protective effect $(1,000 \mathrm{U} / \mathrm{kg}$, $29.2 \% \pm 0.8 \%, P \leq 0.001 ; 2,500 \mathrm{U} / \mathrm{kg}, 28.9 \% \pm 1.4 \%, P \leq 0.01 ; 5,000$ $\mathrm{U} / \mathrm{kg}, 26.0 \% \pm 0.7 \%, P \leq 0.001$; Figure $1 \mathrm{C})$. These data demonstrate that correcting retinal Epo deficiency during the first phase of

\section{Figure 1}

Epo treatment prevents oxygen-induced retinal vessel loss in a dosedependent manner. (A) Real-time PCR quantification of Epo mRNA expression in age-matched mouse retinas under normoxia or hyperoxia treatment; copy number of Epo mRNA $/ 10^{6}$ copies of cyclophilin A control mRNA at P8, P12, P15, and P17 ( $n=6$ per group). (B) Representative retinal whole-mounts showing area of vaso-obliteration after $18 \mathrm{~h}$ of oxygen exposure and i.p. injection (P6 and P7) of Epo (right) or saline control (left). Original magnification, $\times 5$. (C) Dose response of Epo protection against retinal vaso-obliteration at P8 (with i.p. injections at P6 and P7) (saline, $n=40$; Epo 1,000 U/kg, $n=12 ; 2,500 \mathrm{U} / \mathrm{kg}$, $n=7 ; 5,000 \mathrm{U} / \mathrm{kg}, n=16){ }^{* \star} P \leq 0.01 ;{ }^{* *} P \leq 0.001$.

retinopathy with exogenous Epo protects the postnatal mouse retina from hyperoxia-induced vaso-obliteration.

Early use of Epo prevents pathological retinal angiogenesis. Given that exogenous Epo protected against hyperoxia-induced vessel loss, we next explored whether early treatment with Epo to preserve vessels would thereby prevent pathological angiogenesis in the second phase of retinopathy. At P17, mice treated early with exogenous Epo during the vessel loss phase (5,000 U/kg i.p., P6, P8, P10, and $\mathrm{P} 12)$ had a vaso-obliterated/total retinal area of $3.2 \% \pm 0.4 \%$ compared with $6.4 \% \pm 0.7 \%$ in saline-injected controls $(P \leq 0.001$; Figure 2 , A and B), an approximately $50 \%$ reduction in nonvascularized retina. Moreover, P17 pups treated with Epo early were significantly protected from pathologic neovascularization $(5.1 \% \pm 0.7 \%)$ compared with PBS control (7.2\% $\pm 0.7 \% ; P \leq 0.02$; Figure 2, A and C), an approximately $30 \%$ suppression. In contrast, i.p. injection of the same dose of Epo after return to room air during the neovascular phase (P14, P15, and P16), when endogenous retinal Epo expression is elevated (Figure 1A), did not decrease the area of avascular retina (Epo $7.6 \% \pm 1.2 \%$ compared with PBS $6.3 \% \pm 0.8 \%$, NS; Figure 2 , D and $\mathrm{E}$ ) or protect against neovascularization (Epo $7.9 \% \pm 1.4 \%$ versus PBS 7.2\% $\pm 1.0 \%$, NS; Figure 2, D and F). Our data suggest that exogenous Epo treatment during the first phase of retinopathy protects the retina from vessel loss and pathological proliferation, whereas using Epo during the second phase is not protective, with a trend toward worsening retinopathy.

Epo protects retinal neurons from apoptosis. In addition to the protection of the retinal vasculature, we also examined the protective effect of Epo on retinal neurons during hyperoxia-induced vessel loss. Epo protects retinal neurons from light-induced or ischemic injury (14-16). In our study, at P17 after induction of oxygeninduced retinopathy, retinal cross-sections from mice treated with Epo (5,000 U/kg i.p., P6, P8, P10, and P12) had significantly less neuronal apoptosis (as shown with TUNEL staining) compared with PBS controls $(P \leq 0.05)$ (Figure $3, \mathrm{~A}$ and $\mathrm{B})$. The same dose of Epo also inhibited caspase-3/-7 activity by approximately $20 \%$ in retinal homogenates from P17 mice (Figure 3C), suggesting that Epo prevents retinal neuronal apoptosis through inhibition of caspase, a proapoptotic enzyme. These data indicate that early exogenous Epo treatment is beneficial not only for retinal vessel survival but also for retinal neuron survival. This may be attributable in part to improved vascular stability and normalization and consequently less retinal ischemia between P12-P17.

Epo increases retinal survival and stability through activation of Epo receptors via $N F-\kappa B$. Epo's effect on retinal vasculature is likely to be multifactorial, acting both locally by preventing apoptosis of retinal vessels and neurons and systemically by mobilizing bone marrow-derived cells to populate injured retinal vessels. We first exam- 

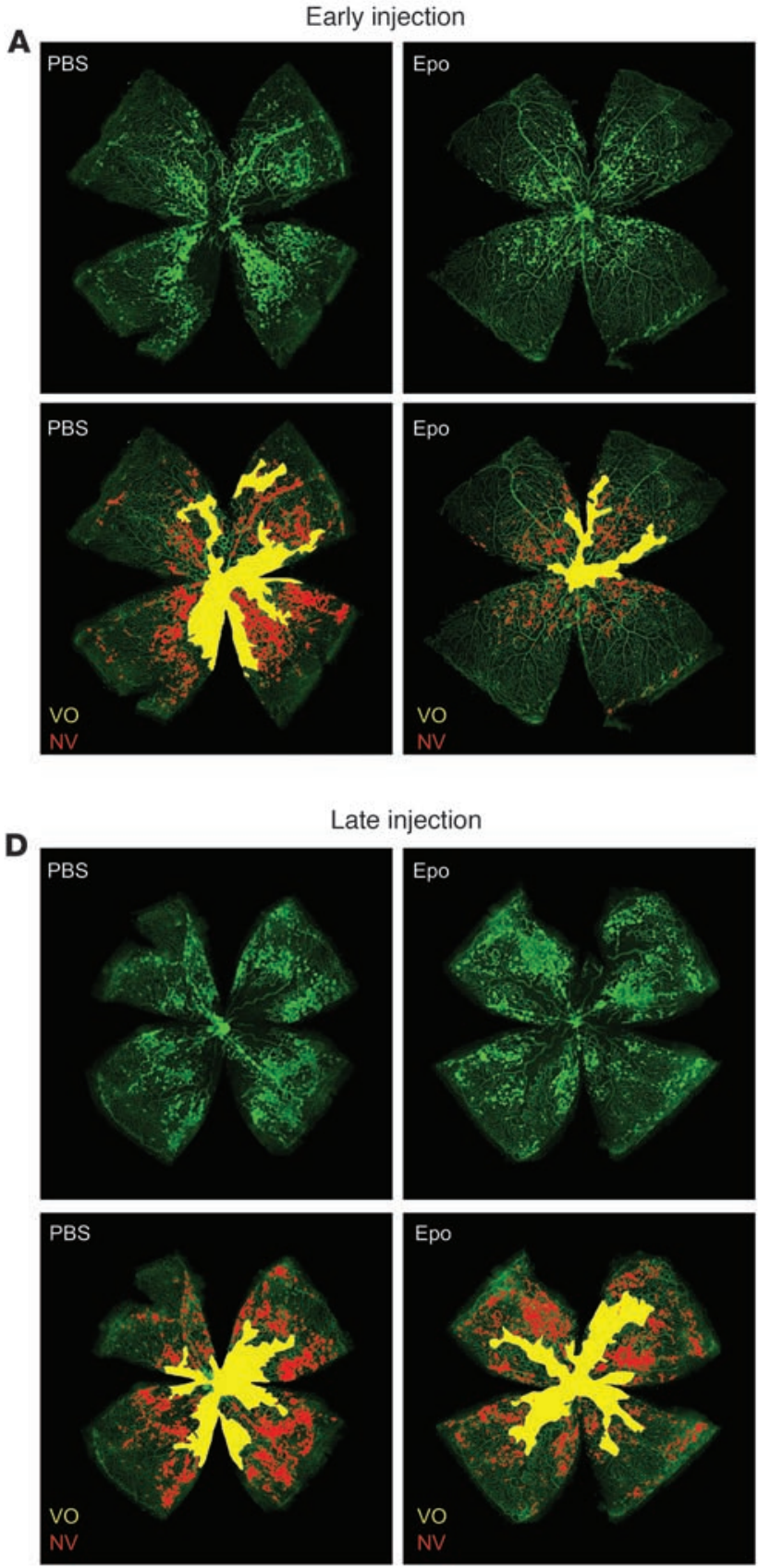
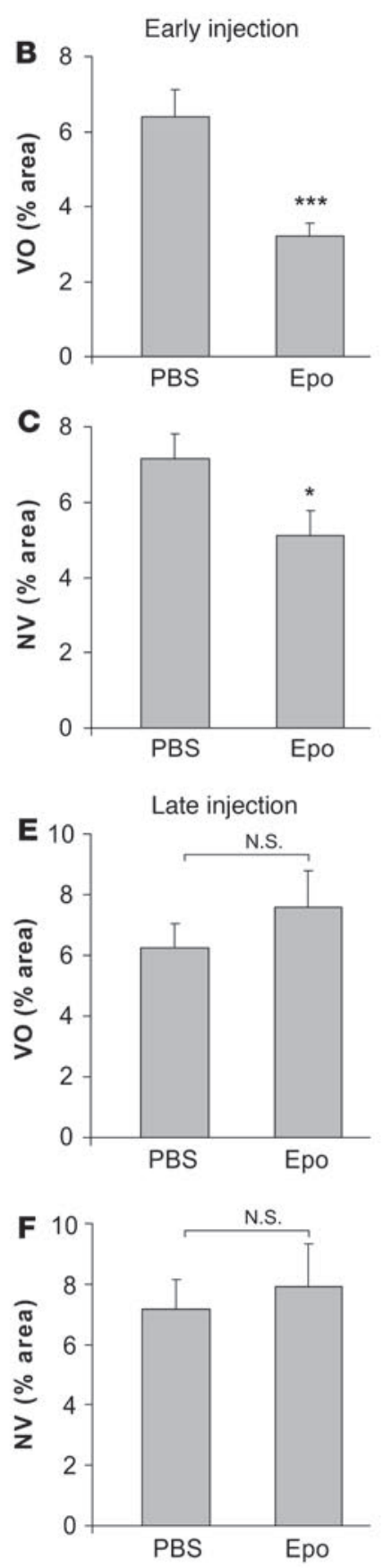

\section{Figure 2}

Early use of Epo protects against retinopathy with reduction in vaso-obliteration and neovascularization; in contrast, late use of Epo is not protective. Retinas were whole-mounted at P17 after oxygen-induced retinopathy, showing both vaso-obliteration (VO) and neovascularization (NV). (A) Representative retina whole-mount with PBS control (left) or early Epo injections (right) (i.p., 5,000 U/kg, P6, P8, P10, and P12). Areas of vaso-obliteration (yellow) and neovascularization (red) were quantified. Original magnification, $\times 5$. (B) Vaso-obliteration $\left({ }^{* * *} P \leq 0.001\right)$ and $(\mathbf{C})$ neovascularization $\left({ }^{*} P \leq 0.02\right)$ in the mice with early Epo treatment at P17 (PBS, $n=40$; Epo, $n=23$ ). (D) Representative retina wholemount with PBS control (left) or late Epo injections (right) (i.p., 5,000 U/kg, P14, P15, and P16) with areas of vaso-obliteration (yellow) and neovascularization (red). Original magnification, $\times 5$. (E) Vaso-obliteration and (F) neovascularization in the mice with late Epo treatment (PBS, $n=34$; Epo, $n=15$ ). ined whether Epo receptors were present in the retina. Epo can activate the Epo receptor as well as a newly discovered $\beta$-common receptor, which is also a coreceptor for colony-stimulating factor. These 2 Epo receptors can form a heterotrimer to signal through several signal transduction cascades to inhibit apoptosis (17). We found that Epo localizes primarily to the inner retina in normal P8 mice and colocalizes with retinal vessels (Figure 4A), suggesting that Epo is an endogenous cytoprotective factor. Epo receptor also colocalizes with retinal vessels in P8 retina and is located predominantly in the inner retina (Supplemental Figure 1; supplemental material available online with this article; doi:10.1172/ JCI33813DS1). Previous studies examining Epo receptor localiza- tion in the retina have been contradictory. Localization has been reported in the ganglion cell layer (16) and in the photoreceptors (14). This inconsistency might partially reflect the finding that Epo receptor antibodies are unreliable for immunohistochemistry (18). To overcome antibody nonspecificity, we used laser capture microdissection of retinal layers and vessels with quantitative realtime RT-PCR to localize mRNA expression of Epo and Epo receptors in the retina (Figure 4B). Pecam expression is highly elevated in microdissected vessel cells, confirming enrichment of vessels (Figure 4C). Epo mRNA is expressed most abundantly in the ganglion cell layer. This pattern is similar to Vegf mRNA expression in the retina (19). Epo receptor is expressed throughout the retina 

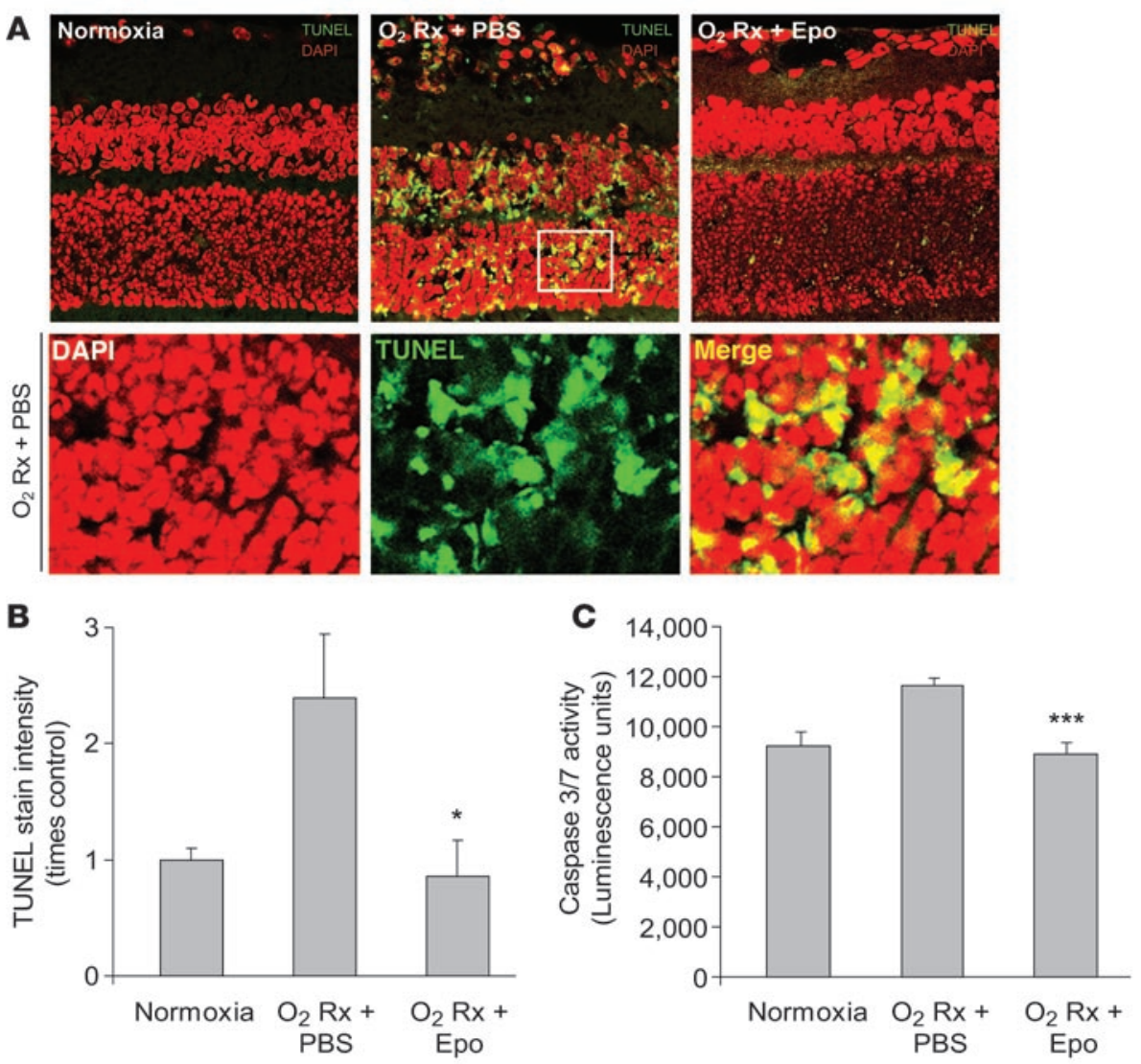

\section{Figure 3}

Early Epo treatment protects retinal neurons from hypoxia-induced apoptosis. (A) TUNEL (green) and DAPI (red) staining of retinal cross-sections from $\mathrm{P} 17$ normoxia mice and oxygen-treated mice with early Epo injection (i.p., 5,000 U/kg, at P6, P8, $\mathrm{P} 10$, and P12) or PBS control. A portion of the image from P17 oxygen-treated PBS control retina was enlarged (white frame) in lower panels. Original magnification, $\times 40$. (B) Quantification of the fluorescence intensity of TUNEL stain in P17 normoxia mice and $\mathrm{P} 17$ hypoxia mice with Epo treatment or PBS control ( $n=3$ per group; $\left.{ }^{\star} P \leq 0.05\right)$. (C) Caspase-3/-7 activity in retinal homogenate isolated from $\mathrm{P} 17$ normoxia mice and $\mathrm{P} 17$ hypoxia mice with Epo treatment or PBS control $(n=6$ per group; ${ }^{\star \star \star} P \leq 0.001$ ). in all retinal layers including vessels, whereas $\beta$-common receptor is expressed most abundantly in ganglion cell layer. These results indicate that Epo might act locally on neurons and vessels through Epo receptors to promote survival. In addition, Epo's protective effect is independent of Vegf, since Epo injections (i.p. P6 and P7) did not change mRNA expressions of Vegf and Vegf receptors (Flt-1, Flk-1, and Nrp1) or hypoxia-inducible factors (Hif1a and Hif $2 a$ ) in the retinas of P8 mice (Figure 4D), consistent with the results from a previous study showing that Epo and Vegf levels in human vitreous are independent (4). The tissue-protective effect of Epo is mediated predominately through NF- $\mathrm{B}$ (17). Using

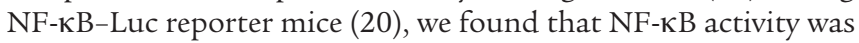
increased approximately $35 \%(P \leq 0.05)$ in the retinas of Epo-treated (i.p., P6 and P7, 5,000 U/kg) versus control mice (Figure 4E), and $\mathrm{NF}-\kappa \mathrm{B}$ was found in all retinal layers (Figure 4F), suggesting that Epo might protect retinal vessels and neurons locally through activation of Epo receptors and modulation of NF- $\mathrm{KB}$.

Epo increases the number of proangiogenic stem cells in retina. In addition to its local effect, exogenous Epo might also act systemically by recruiting bone marrow-derived proangiogenic cells to vessels, since Epo has been reported to increase mobilization of endothelial progenitor cells (EPCs) from bone marrow (21). We found that Epo treatment $(5,000 \mathrm{U} / \mathrm{kg}$, i.p. P6 and P7) increases the number of CD $34^{+}$EPCs per vessel length by approximately $80 \%(P \leq 0.001$; Figure 5, A and B) in the retinas of $\mathrm{P} 8$ mice exposed to oxygen. The same dose of Epo also increases the number of Csf- $1 \mathrm{R}^{+}$microglia in the retina by approximately $30 \%(P \leq 0.005$; Figure $5, \mathrm{C}$ and $\mathrm{D})$. Both EPCs $(22,23)$ and microglia $(24,25)$ are important in the repair of injured retinal vasculature. These results suggest that in addition to acting locally through Epo receptors in the retina, Epo can also promote retinal vascular repair by recruiting bone marrow-derived proangiogenic cells to the retina.

\section{Discussion}

Although retinal Epo is known to be elevated during proliferative retinopathy, and suppression of Epo suppresses neovascularization (4), the role of Epo in the initial process of vessel loss during retinopathy development has not been described. Using exogenous Epo treatment, this study has distinguished the contributions of Epo to each phase of retinopathy in the neonatal mouse and suggests that timing of Epo intervention is critical. Specifically, we found that Epo deficiency in the first phase of retinopathy can contribute to the development of retinopathy, and early Epo supplementation to compensate for the deficiency during the first phase of retinopathy promotes vascular and neuronal survival. Treating with Epo early, prior to, or during vascular loss helps prevent the vessel loss and ischemia, thereby suppressing subsequent retinal neovascularization. Early use of Epo also protects retinal neurons from hypoxia-induced apoptosis, which is consistent with previous studies that have found that Epo can protect retinal ganglion cells and photoreceptors from ischemia or light-induced degeneration (14-16). These findings suggest the important possibility of treating retinopathy patients early with Epo in order to prevent retinal ischemia without provoking neovascularization.

In contrast, late Epo treatment during the neovascularization phase of retinopathy does not protect retinal vasculature, but instead might exacerbate pathological proliferation. Late-phase proliferative DR and ROP are characterized by abnormal blood 

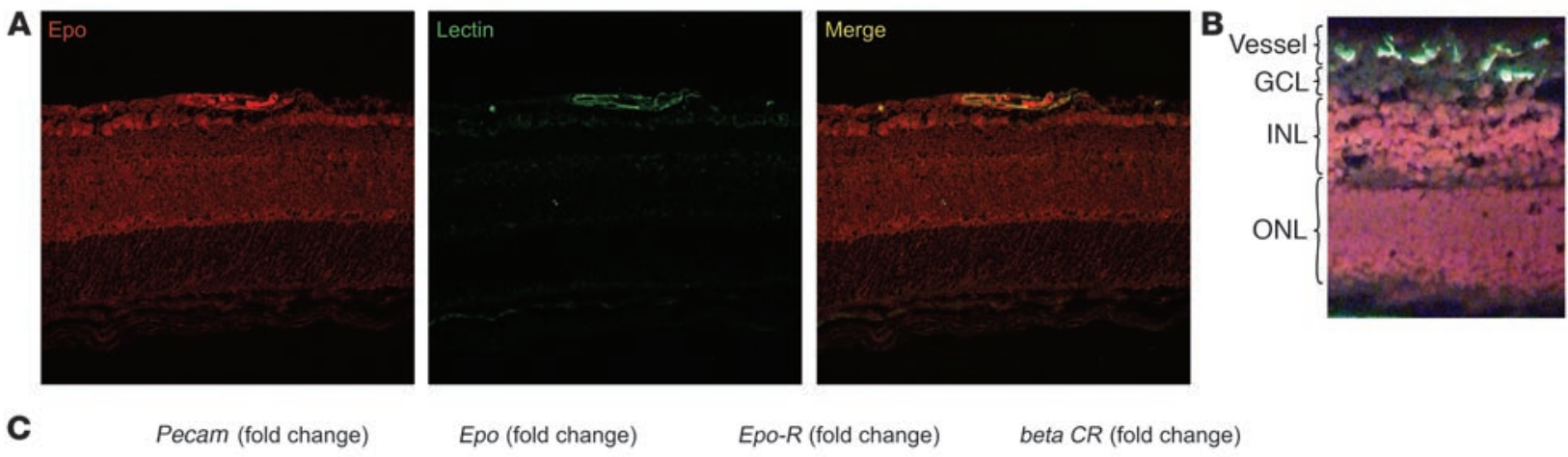

Epo (fold change)
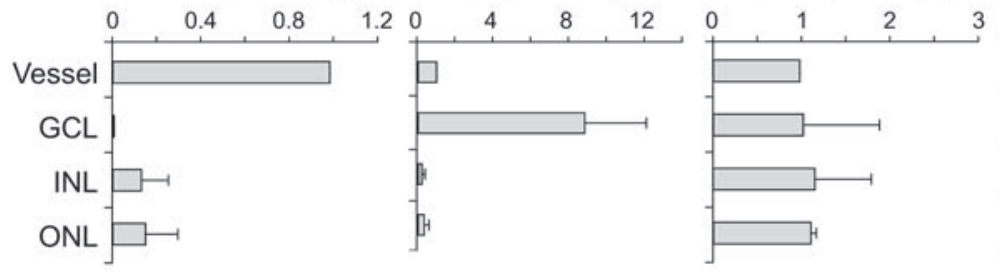

beta $C R$ (fold change)

D

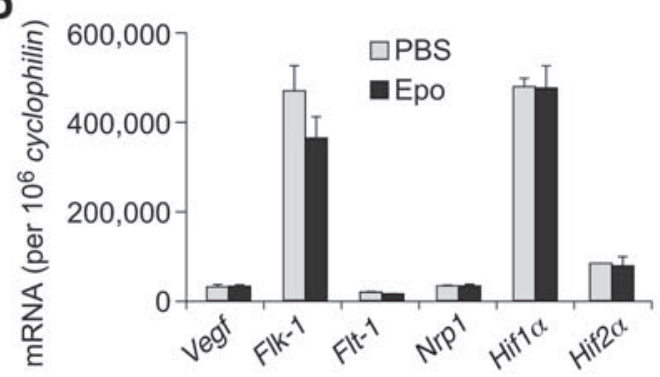

E

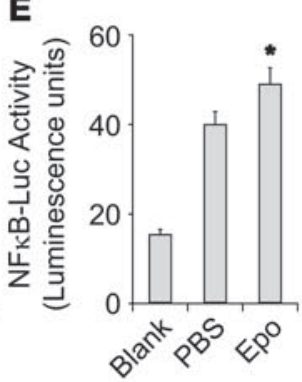

$\begin{array}{llllll}0 & 2 & 4 & 6 & 8 & 10\end{array}$

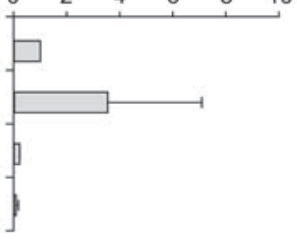

$\mathbf{F}$

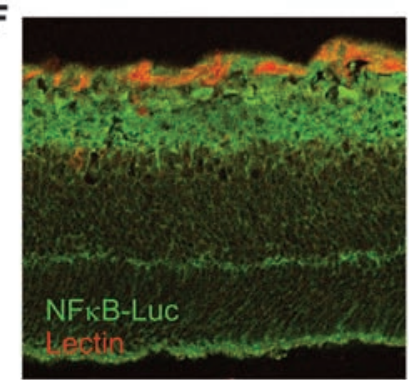

Figure 4

Localization of Epo and Epo receptors and Epo-induced NF-кB activity in the retina. (A) Representative retinal cross-sections from P8 normoxia mouse immunolabeled with Epo antibody (red) and lectin (green). (B) Dehydrated retinal cross-section from P8 normoxia retina stained with lectin (green) and counter-stained with H\&E for laser capture microdissection. (C) mRNA expression of Pecam, Epo, Epo receptor (Epo-R), and $\beta$-common receptor $(\beta C R)$ in laser-captured retinal cell layers $(n=6$ per group). GCL, ganglion cell layer; INL, inner nuclear layer; ONL, outer nuclear layer. (D) Real-time PCR quantification of Vegf and Vegf receptor (Flk-1, Flt-1, Nrp1, Hif1a, and Hif2a) mRNA in retina of mouse littermate with Epo treatment (P6 and P7) or PBS control ( $n=6$ per group). Copy number of mRNA/106 copies cyclophilin A control mRNA were measured at P8 ( $n=8$ per group). (E) P8 oxygen-treated retinas from NF-kB-Luc reporter mice with PBS $(n=6)$ or Epo treatment (i.p., 5,000

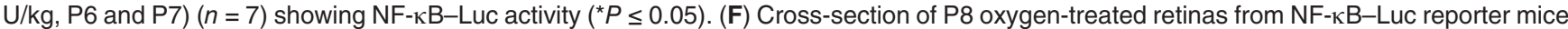
stained with lectin GS-IB4 (red) and luciferase antibody (green) showing NF-kB localization. Original magnification, $\times 40$.

vessel growth in the retina. These leaky vessels can lead to tractional retinal detachment and blindness. In this neovascularization phase, Epo is already elevated, contributing to the pathological retinal angiogenesis. Administrating recombinant Epo to these patients might further precipitate vessel proliferation.

It is likely that Epo protects retinal vessels and neurons through both local and systemic mechanisms. Epo is important for normal vascular development, since depletion of Epo and Epo receptor leads to angiogenic defects and an embryonic lethal phenotype (26). Epo is expressed in the retina suggesting that it is an endogenous retinal survival factor. Epo/Epo receptor systems can increase endothelial cell proliferation and protect vasculature against ischemia and apoptosis $(27,28)$. Epo receptors are present in both retinal vessels and neurons. Therefore they may be activated locally to promote retinal cell survival. Consistent with the cytoprotective effect of Epo through crosstalk of Jak2 and NF-кB signaling (29), we found that exogenous Epo increases the activity of NF- $\mathrm{B}$, suggesting that Epo might activate local Epo receptors and NF- $\mathrm{B}$ signaling, which then initiate antiapoptotic response locally in the retina.

Epo can also stimulate mobilization of bone marrow-derived stem cells into the circulation to promote neonatal neovascularization $(21,30)$. Our study found that Epo can stimulate recruitment of bone marrow EPCs into the retina, which have been shown to differentiate into endothelial cells and revascularize injured retinal vasculature $(22,23)$. Epo can also increase the number of proangiogenic microglia/macrophages in the retina, which is crucial to retinal vascular growth and repair $(24,25)$. These observations suggest a systemic impact of Epo intervention and might help partially explain the adverse cardiovascular effect of Epo intervention if used during a period when the angiogenic response has already started.

Although Epo and Vegf exhibit similar angiogenic potential (31), Epo production appears to be independent of Vegf (4). Our study also indicates that exogenous Epo does not change Vegf or Vegf receptor expression, suggesting that Epo may be additive to anti-VEGF therapy. 

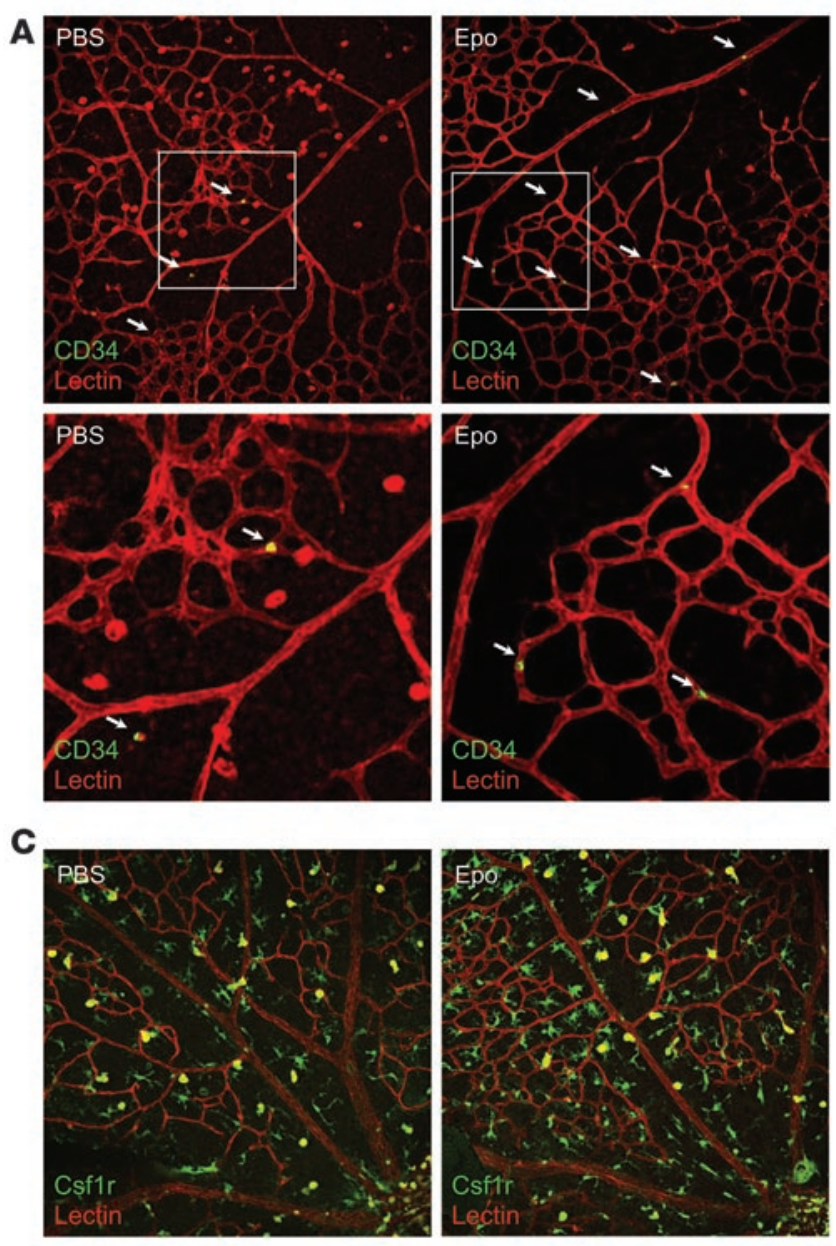
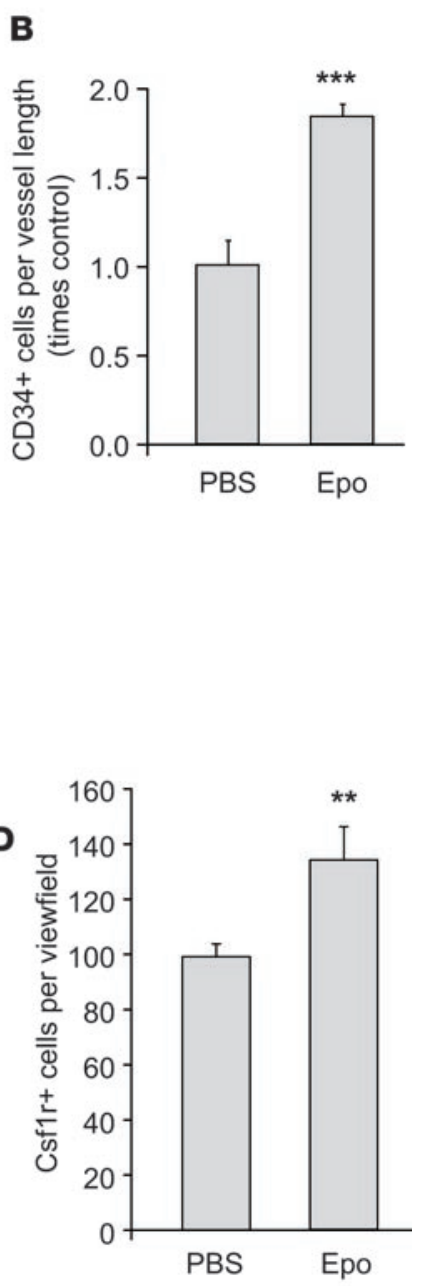

\section{Figure 5}

Epo-induced increase of EPC and microglia numbers in the retina. (A) Representative images of retinal flat mount from P8 oxygen-treated mice with Epo injection (i.p., 5,000 U/kg, P6 and P7) or PBS control stained with CD34 antibody (green) and lectin GS-IB4 (red), with a portion of the image enlarged (white frames). White arrows indicate CD $34^{+}$cells. (B) Mean number of EPCs (CD34+ cells) per vessel length in Epo treated compared with control retina ( $n=6$ per group; $\left.{ }^{\star * *} P \leq 0.001\right)$. (C) Representative images of retinal flat mount from P8 oxygen-treated mice with Epo injection (i.p., 5,000 U/kg, P6 and P7) or PBS control stained with Csf-1R antibody (green) and lectin GS-IB4 (red). (D) Mean number of microglia (Csf-1 $\mathrm{R}^{+}$cells) in Epo-treated versus control retinas $\left(n=10\right.$ per group; $\left.{ }^{\star \star} P \leq 0.005\right)$. Original magnification, $\times 20$.
In summary, these results suggest that caution must be exercised when prescribing Epo to patients with proliferative retinopathy. The current findings encourage screening diabetic and premature patients for retinopathy and prescribing Epo only before the proliferative stage develops. The effect of Epo on angiogenesis is likely to be important not only for retinopathy but for other pathologies as well. Higher doses of Epo have been linked with increased risk of cardiovascular disease and tumor growth (5-8). Without careful dosing and timing, Epo treatment might exacerbate pathological angiogenesis.

\section{Methods}

Animals. These studies adhered to the Association for Research in Vision and Ophthalmology (ARVO) Statement for the Use of Animals in Ophthalmic and Vision Research and were approved by the Children's Hospital Boston Animal Care and Use Committee. Unless otherwise indicated, 129S6/SvEv mice (129SVE; Taconic) were used for the study. The NF-KB-Luc reporter mice (stock no. 006100; JAX) were made using the pBIIX-luciferase targeting construct with 2 copies of the NF-KB binding sites from the $\kappa$-light chain intronic enhancer in front of a minimal fos promoter, as described previously (20).

$\mathrm{O}_{2}$-induced retinopathy (vessel loss and pathological neovascularization). To induce vessel loss, mice with their nursing mother were exposed to $75 \%$ oxygen from P7 to P12 (9). Retinal vessel loss was assessed at P8, $18 \mathrm{~h}$ after oxygen exposure, when the vessel loss is greatest. Retinal neovasculariza- tion was evaluated at P17, 5 days after return to room air, when the neovascular response is greatest.

FITC-dextran perfusion and retina flat mount. P8 mice were anesthetized with Avertin (Sigma-Aldrich) and sacrificed by intracardiac perfusion with $4 \%$ paraformaldehyde and FITC-dextran (molecular weight $2 \times 10^{6}$ ) in PBS (9). Eyes were enucleated and fixed in $4 \%$ paraformaldehyde for $1 \mathrm{~h}$ at room temperature. The retinas were dissected and whole-mounted onto Superfrost/Plus microscope slides (catalog no. 12-550-15; Fisher Scientific) with the photoreceptor side down and imbedded in SlowFade Antifade reagent (catalog no. S2828; Invitrogen).

Retina neovascularization after oxygen-induced vessel loss. At P17, mice were given lethal doses of Avertin, and eyes were fixed in $4 \%$ paraformaldehyde for $1 \mathrm{~h}$. Retinas were isolated and stained overnight at $23^{\circ} \mathrm{C}$ with fluoresceinated Griffonia Bandeiraea Simplicifolia Isolectin B4 (Alexa Fluor 488-conjugated I21411 or Alexa Fluor 594-conjugated I21413; Molecular Probes; 1:100 dilution) in $1 \mathrm{mM} \mathrm{CaCl}_{2}$ in PBS. Following $2 \mathrm{~h}$ of washes, retinas were whole-mounted.

Quantification of vaso-obliteration and retinal neovascularization. Quantification of vaso-obliteration and neovascularization were carried out as described previously $(32,33)$. Images of each of the 4 quadrants of wholemounted retina were taken at $\times 5$ magnification on a Leica SP 2 confocal microscope and imported into Adobe Photoshop. Retinal segments were merged to produce an image of the entire retina. Vaso-obliteration and neovascular tuft formation were quantified by comparing the number of pixels in the affected areas with the total number of pixels in the retina. 
Percentages of vaso-obliteration and neovascularization from mouse retinas were compared with values for retinas from age-matched control mice with identical oxygen conditions. Evaluation was done blind to the identity of the sample. $n$ is number of eyes quantified.

Recombinant Epo injection. For evaluation of vessel loss, at P6 and P7, varying doses $(1,000 \mathrm{U} / \mathrm{kg}, 2,500 \mathrm{U} / \mathrm{kg}$, and 5,000 U/kg) of Epo (Epoetin alfa) in $20 \mu \mathrm{l}$ PBS were injected i.p. Control PBS was injected into littermates. After $18 \mathrm{~h} \mathrm{O}_{2}$ exposure, eyes from P8 mice were perfused with FITC-dextran, and retinas were dissected and whole-mounted for analysis of vessel loss. For analysis of neovascularization, Epo $(5,000 \mathrm{U} / \mathrm{kg})$ was injected i.p. either prior to or during oxygen treatment (at P6, P8, P10, and P12) or after returning to room air (at P14, P15, and P16). Retinal neovascularization was analyzed on lectin-stained P17 retinal flat mounts.

RNA isolation and cDNA preparation. Total RNA was extracted using an RNeasy kit (QIAGEN) from the retinas of 1 mouse from each of 6 litters and then pooled to reduce biologic variability. Retinas from each time point were lysed in guanidinium isothiocyanate lysis buffer following the manufacturer's instructions, and RNA was suspended in diethyl pyrocarbonate-treated $\mathrm{H}_{2} \mathrm{O}$. To generate cDNA, $1 \mu \mathrm{g}$ total RNA was treated with DNase I (Ambion Inc.) to remove any contaminating genomic DNA. The DNase-treated RNA (100 ng) was then converted into cDNA using murine leukemia virus reverse transcriptase (Invitrogen). All cDNA samples were aliquoted and stored at $-80^{\circ} \mathrm{C}$.

Quantitative real-time PCR analysis of gene expression. PCR primers targeting Epo, Epo receptor, $\beta$-common receptor, Pecam, and an unchanging control gene, cyclophilin A, were designed using Primer Express software (Applied BioSystems). We used 3 methods to analyze primer and probe sequences for specificity of gene detection. First, only primer and probe sequences that specifically detected the sequence of choice, as determined by means of the NCBI Blast module, were used. Second, amplicons generated during the PCR reaction were analyzed using the first derivative primer melting curve software supplied by Applied BioSystems. This analysis determined the presence of amplicons on the basis of their specific melting point temperatures. Third, amplicons generated during the PCR reaction were gel purified and sequenced (by the Children's Hospital Boston Core Sequencing Facility) to confirm the selection of the desired sequence. Quantitative analysis of gene expression was generated using an ABI Prism 7700 Sequence Detection System (TaqMan) and the SYBR Green Master mix kit. Standard curves for each gene were plotted with quantified cDNA template during each real-time PCR reaction. Each target gene mRNA copy number was then normalized to $10^{6}$ copies of cyclophilin A control.

Epo $m R N A$ expression during oxygen-induced retinopathy. Retinas were isolated at P8, P12, P15, and P17 ( $n=6$ per time point) from hyperoxia and normoxia control C57BL/ 6 mice. Retinas were combined, and RNA was isolated and converted to cDNA. Epo mRNA expression was compared with cyclophilin expression after quantitative RT-PCR.

TUNEL staining. Epo-treated (i.p. 5,000 U/kg body weight, P6, P8, P10, and P12) or control P17 mice were anesthetized, and eyes were enucleated and fixed in $4 \%$ paraformaldehyde $(1 \mathrm{~h})$. Tissue was then cryoprotected in $30 \%$ sucrose overnight, embedded in OCT (Fisher Scientific Co.), and sectioned with a cryostat at $14 \mu \mathrm{m}$. Sequential vertical sections cut through the center of the eye were labeled by TUNEL with Roche in situ cell death detection kit (catalog no. 11684795910; Roche Applied Science) followed by DAPI staining (catalog no. H1200; Vector Laboratory). DAPI staining was displayed with a red LUT to better visualize colocalization with TUNEL staining (green fluorescence). Retinas were examined using fluorescence microscope, and TUNEL-positive fluorescent intensity was scored.

Caspase activity assay. Epo-treated (i.p. 5,000 U/kg body weight, P6, P8, $\mathrm{P} 10$, and $\mathrm{P} 12$ ) or control P17 mice were anesthetized, and retinas were isolated and homogenized in Glow Lysis Buffer (catalog no. E2661; Promega).
Retinal homogenate $(50 \mu \mathrm{g})$ was analyzed for caspase-3/-7 activity using Promega Caspase-Glo 3/7 Assay system (G8091; Promega).

Immunohistochemical staining. Eyes fixed in $4 \%$ paraformaldehyde and frozen in OCT were cut into 14- $\mu \mathrm{m}$ sections, rinsed with PBS, and then blocked in PBS with $0.5 \%$ Triton X-100 and $5 \%$ goat serum. The sections were stained with primary antibodies against Epo and Epo receptor (Santa Cruz Biotechnology Inc.), followed by anti-rabbit secondary antibodies (Alexa Fluor 594; Molecular Probes) and thereafter FITC-conjugated Griffonia Bandeiraea Simplicifolia Isolectin B4. For whole-mount immunohistochemical staining, retinas fixed in $4 \%$ paraformaldehyde for $1 \mathrm{~h}$ were rinsed in PBS, permeabilized overnight at $4{ }^{\circ} \mathrm{C}$ with $0.5 \%$ Triton X-100 (catalog no. T-8787; Sigma-Aldrich) in PBS, and stained with Isolectin $\mathrm{B} 4$, as described above. Retinal whole-mounts were prepared as described previously and visualized with a Leica SP2 confocal microscope. Retinal microglia/macrophages were visualized in retinas from mice with Csf1r-driven GFP (stock no. 005070; JAX). Fluorescence was enhanced with rabbit antibodies against GFP (catalog no. A11122; Invitrogen) and FITClabeled secondary antibodies (catalog no. F6005; Sigma-Aldrich). Microglia density was quantified by collecting confocal images of the superficial central retina using a $\times 20$ objective lens, including a quadrant of the optic nerve head as a point of reference. For visualization of retinal EPCs, retinas were dissected after PBS perfusion to remove circulating blood cells, and CD34-FITC antibodies (Miltenyi Biotechnology) were used according to the manufacturer's recommendations. EPC density was quantified by collecting confocal images of the superficial retina in the center of each retina quadrant using a $\times 20$ objective lens. Mean number of EPCs was normalized against vessel length measured with Northern Eclipse software (Empix Imaging), and the results were reported as fold change compared with fold change of controls. Evaluation was done blind to the identity of the sample, and resulting values from each image were averaged. $n$ is the number of eyes quantified.

Laser capture microdissection of retinal layers. Eyes were embedded in OCT immediately following enucleation, cut into 8 - $\mu \mathrm{m}$ sections, and collected on plain, uncharged, RNase-free slides. Sections were stained with isolectin, counterstained with $\mathrm{H} \& \mathrm{E}$, and dehydrated with $50 \%, 70 \%, 95 \%$, and $100 \%$ ethanol and xylene. Four retinal layers (vessel layer, ganglion cell layer, inner nuclear layer, and outer nuclear layer) were microdissected with an Arcturus PixCell IIe LCM System. RNA was extracted from microdissected tissues with a Picopure RNA isolation kit (Molecular Devices), and real-time PCR was performed.

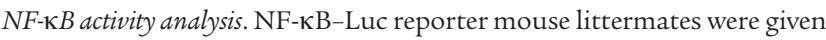
PBS or Epo treatment (i.p. 5,000 U/kg body weight) on P6 and P7. After $18 \mathrm{~h}$ oxygen exposure, P8 pups were sacrificed and retinas were isolated and homogenized in Glow Lysis Buffer (catalog no. E2661; Promega). Retinal homogenate $(50 \mu \mathrm{g})$ was analyzed for luciferase activity using Promega Bright-Glo Luciferase Assay system (catalog no. E2610). Additionally, retinal cross-section of NF-KB-Luc reporter mouse were taken at P8 and stained with rabbit antibodies to luciferase (catalog no. ab21176; Abcam).

Statistics. Results are presented as mean \pm SEM for animal studies and mean \pm SD for the non-animal studies. ANOVA with $\alpha=0.05$ was used for processing the data. A 2 -sample $t$ test was used as a post test unless otherwise indicated.

\section{Acknowledgments}

We would like to thank Nan Liu and Elke A. Pravda for excellent technical help in performing experiments and operating the microscope. This research was generously supported by the V. Kann Rasmussen Foundation, NIH grants EY008670, EY017017, and EY14811 (to L.E.H. Smith) and 5 T32 EY07145 and 1 F32 EY017789-01 (to K.M. Connor), and Children's Hospital Boston 
Mental Retardation and Developmental Disabilities Research Center grant P01 HD18655 (to L.E.H. Smith). We thank the Juvenile Diabetes Foundation for fellowship support (to J. Chen). We appreciate support from the Research to Prevent Blindness Lew Wasserman Merit Award (to L.E.H. Smith). The sponsors had no role in the design or conduct of the study, in the collection, analysis, and interpretation of data, or in the preparation, review, or approval of the manuscript.

1. Gariano, R.F., and Gardner, T.W. 2005. Retinal angiogenesis in development and disease. Nature. 438:960-966.

2. Sasaki, R. 2003. Pleiotropic functions of erythropoietin. Intern. Med. 42:142-149.

3. Katsura, Y., et al. 2005. Erythropoietin is highly elevated in vitreous fluid of patients with proliferative diabetic retinopathy. Diabetes Care. 28:2252-2254.

4. Watanabe, D., et al. 2005. Erythropoietin as a retinal angiogenic factor in proliferative diabetic retinopathy. N. Engl. J. Med. 353:782-792.

5. Singh, A.K., et al. 2006. Correction of anemia with epoetin alfa in chronic kidney disease. N. Engl. J. Med. 355:2085-2098.

6. Wright, J.R., et al. 2007. Randomized, double-blind, placebo-controlled trial of erythropoietin in nonsmall-cell lung cancer with disease-related anemia. J. Clin. Oncol. 25:1027-1032.

7. Bohlius, J., et al. 2005. Recombinant human erythropoietin and overall survival in cancer patients: results of a comprehensive meta-analysis. J. Natl. Cancer Inst. 97:489-498.

8. Bohlius, J., et al. 2006. Recombinant human erythropoietins and cancer patients: updated meta-analysis of 57 studies including 9353 patients. J. Natl. Cancer Inst. 98:708-714.

9. Smith, L.E., et al. 1994. Oxygen-induced retinopathy in the mouse. Invest. Ophthalmol. Vis. Sci. 35:101-111.

10. Ribatti, D., Vacca, A., Roccaro, A.M., Crivellato, E., and Presta, M. 2003. Erythropoietin as an angiogenic factor. Eur. J. Clin. Invest. 33:891-896.

11. Li, F., Chong, Z.Z., and Maiese, K. 2004. Erythropoietin on a tightrope: balancing neuronal and vascular protection between intrinsic and extrinsic pathways. Neurosignals. 13:265-289.

12. Thomas, M.C. 2006. The high prevalence of anemia in diabetes is linked to functional erythropoietin deficiency. Semin. Nephrol. 26:275-282.
Received for publication September 4, 2007, and accepted in revised form November 28, 2007.

Address correspondence to: Lois E.H. Smith, Department of Ophthalmology, Children's Hospital Boston, Harvard Medical School, 300 Longwood Ave., Boston, Massachusetts 02115, USA. Phone: (617) 355-8531; Fax: (617) 730-0328; E-mail: lois.smith@ childrens.harvard.edu.
13. Thomas, M.C. 2007. Anemia in diabetes: marker or mediator of microvascular disease? Nat. Clin. Pract. Nephrol. 3:20-30.

14. Grimm, C., et al. 2002. HIF-1-induced erythropoietin in the hypoxic retina protects against lightinduced retinal degeneration. Nat. Med. 8:718-724.

15. Weishaupt, J.H., et al. 2004. Effect of erythropoietin axotomy-induced apoptosis in rat retinal ganglion cells. Invest. Ophthalmol. Vis. Sci. 45:1514-1522.

16. Kilic, U., et al. 2005. Erythropoietin protects from axotomy-induced degeneration of retinal ganglion cells by activating ERK-1/-2. FASEB J. 19:249-251.

17. Brines, M., and Cerami, A. 2005. Emerging biologi$\mathrm{cal}$ roles for erythropoietin in the nervous system. Nat. Rev. Neurosci. 6:484-494.

18. Elliott, S., et al. 2006. Anti-Epo receptor antibodies do not predict Epo receptor expression. Blood. 107:1892-1895.

19. Pierce, E.A., Avery, R.L., Foley, E.D., Aiello, L.P., and Smith, L.E. 1995. Vascular endothelial growth factor/vascular permeability factor expression in a mouse model of retinal neovascularization. Proc. Natl. Acad. Sci. U. S. A. 92:905-909.

20. Millet, I., et al. 2000. Inhibition of NF-kappaB activity and enhancement of apoptosis by the neuropeptide calcitonin gene-related peptide. J. Biol. Chem. 275:15114-15121.

21. Heeschen, C., et al. 2003. Erythropoietin is a potent physiologic stimulus for endothelial progenitor cell mobilization. Blood. 102:1340-1346.

22. Grant, M.B., et al. 2002. Adult hematopoietic stem cells provide functional hemangioblast activity during retinal neovascularization. Nat. Med. 8:607-612.

23. Otani, A., et al. 2002. Bone marrow-derived stem cells target retinal astrocytes and can promote or inhibit retinal angiogenesis. Nat. Med. 8:1004-1010.

24. Ritter, M.R., et al. 2006. Myeloid progenitors differentiate into microglia and promote vascular repair in a model of ischemic retinopathy. J. Clin. Invest. 116:3266-3276.

25. Checchin, D., Sennlaub, F., Levavasseur, E., Leduc, M., and Chemtob, S. 2006. Potential role of microglia in retinal blood vessel formation. Invest. Ophthalmol. Vis. Sci. 47:3595-3602.

26. Kertesz, N., Wu, J., Chen, T.H., Sucov, H.M., and $\mathrm{Wu}, \mathrm{H} .2004$. The role of erythropoietin in regulating angiogenesis. Dev. Biol. 276:101-110.

27. Anagnostou, A., Lee, E.S., Kessimian, N., Levinson, R., and Steiner, M. 1990. Erythropoietin has a mitogenic and positive chemotactic effect on endothelial cells. Proc. Natl. Acad. Sci. U. S. A. 87:5978-5982.

28. Chong, Z.Z., Kang, J.Q., and Maiese, K. 2002. Erythropoietin is a novel vascular protectant through activation of Akt 1 and mitochondrial modulation of cysteine proteases. Circulation. 106:2973-2979.

29. Digicaylioglu, M., and Lipton, S.A. 2001. Erythropoietin-mediated neuroprotection involves crosstalk between Jak2 and NF-kappaB signalling cascades. Nature. 412:641-647.

30. Bahlmann, F.H., et al. 2004. Erythropoietin regulates endothelial progenitor cells. Blood. 103:921-926.

31. Jaquet, K., Krause, K., Tawakol-Khodai, M., Geidel, S., and Kuck, K.H. 2002. Erythropoietin and VEGF exhibit equal angiogenic potential. Microvasc. Res. 64:326-333.

32. Banin, E., et al. 2006. T2-TrpRS inhibits preretinal neovascularization and enhances physiological vascular regrowth in OIR as assessed by a new method of quantification. Invest. Ophthalmol. Vis. Sci. 47:2125-2134.

33. Gebarowska, D., et al. 2002. Synthetic peptides interacting with the $67-\mathrm{kd}$ laminin receptor can reduce retinal ischemia and inhibit hypoxiainduced retinal neovascularization. Am. J. Pathol. 160:307-313. 